\title{
Special Spline Approximation for the Solution of the Non-Stationary 3-D Mass Transfer Problem
}

\author{
Ilmārs Kangro \\ Faculty of Engineering \\ Rezekne Academy of Technologies \\ Rezekne, Latvia \\ ilmars.kangro@rta.lv
}

\author{
Harijs Kalis \\ Institute of Mathematics and \\ Computer sciences \\ University of Latvia \\ Riga, Latvia \\ harijs.kalis@lu.lv \\ Edmunds Teirumnieks \\ Faculty of Engineering) \\ Rezekne Academy of Technologies \\ Rezekne, Latvia \\ Edmunds.Teirumnieks@rta.lv
}

\author{
Ērika Teirumnieka \\ Faculty of Engineering) \\ Rezekne Academy of Technologies \\ Rezekne, Latvia \\ Erika.Teirumnieka@rta.lv
}

\begin{abstract}
In this paper we consider the conservative averaging method (CAM) with special spline approximation for solving the non-stationary 3-D mass transfer problem. The special hyperbolic type spline, which interpolates the middle integral values of piece-wise smooth function is used. With the help of these splines the initial-boundary value problem (IBVP) of mathematical physics in 3-D domain with respect to one coordinate is reduced to problems for system of equations in 2-D domain. This procedure allows reduce also the 2-D problem to a 1-D problem and thus the solution of the approximated problem can be obtained analytically. The accuracy of the approximated solution for the special 1D IBVP is compared with the exact solution of the studied problem obtained with the Fourier series method. The numerical solution is compared with the spline solution. The above-mentioned method has extensive physical applications, related to mass and heat transfer problems in 3-D domains.
\end{abstract}

Keywords - conservative averaging method, 3-D mass transfer problem, hyperbolic type splines, analytical solution

\section{INTRODUCTION}

The task of sufficient accuracy numerical simulation of quickly solution 3-D problems for mathematical physics is important in known areas of the applied sciences, for example, the calculation of the metal concentration in peat blocks. The metals distribution in peat layer's blocks have been modelled in [3], [4].
A.Buikis had considered the conservative averaging method (CAM) with the integral parabolic type splines for mathematical simulation of the mass transfer processes in multilayered underground systems [1].

The conservative averaging method has been applied also in a technical sphere, modelling the heat distribution in the 3-D area of the automotive fuse [5]. Cylindrical mathematical model of automotive fuse due to characterize the heat-up process in the fuse is described by partial differential equations of the transient heat conduction. CAM with integral parabolic type splines has been used to get the approximated solution of studied problem with analytical formulas [6].

In the present paper CAM using the special hyperbolic type splines is developed. With the help of these splines the IBVP in 3-D domain with respect to one coordinate is reduced to 2-D and 1-D problems. These splines in every direction of averaging contain parameters, where being based on CAM it can be chosen so that the error of the solution is decreasing.

The accuracy of the approximated solution for the special 1-D problem is compared with the exact solution of the studied problem obtained by the Fourier series method. The best values of the parameters (for minimizing the error of the solution) can be obtained with the different orientation of the averaging method, that is, applying the averaging method in the $x$ and $y$ directions respectively. 
In the limit case when the hyperbolic type spline parameters tend to zero we get the integral parabolic spline, developed from A. Buikis [1].

\section{MATERIALS AND METHODS}

\section{THE MATHEMATICAL MODEL}

We will find the distribution of concentrations $c(x, y, z)$ at the point $(x, y, z) \in \Omega$ and at the time $t$ from the following 3-D initial-boundary value mass transfer problem for partial differential equation (PDE) (1):

$$
\left\{\begin{array}{l}
\frac{\partial}{\partial x}\left(D_{x} \frac{\partial c}{\partial x}\right)+\frac{\partial}{\partial y}\left(D_{y} \frac{\partial c}{\partial y}\right)+\frac{\partial}{\partial z}\left(D_{z} \frac{\partial c}{\partial z}\right)=\frac{\partial c}{\partial t}, \\
x \in\left(0, L_{x}\right), y \in\left(0, L_{y}\right), z \in\left(0, L_{z}\right), t \in\left(0, t_{f}\right), \\
\frac{\partial c(0, y, z, t)}{\partial x}=\frac{\partial c(x, 0, z, t)}{\partial y}=\frac{\partial c(x, y, 0, t)}{\partial z}=0, \\
D_{x} \frac{\partial c\left(L_{x}, y, z, t\right)}{\partial x}+\alpha_{x}\left(c\left(L_{x}, y, z, t\right)-c_{a x}\right)=0, \\
D_{y} \frac{\partial c\left(x, L_{y}, z, t\right)}{\partial y}+\alpha_{y}\left(c\left(x, L_{y}, z, t\right)-c_{a y}\right)=0, \\
D_{z} \frac{\partial c\left(x, y, L_{z}, t\right)}{\partial z}+\alpha_{z}\left(c\left(x, y, L_{z}, t\right)-c_{a z}\right)=0, \\
c(x, y, z, 0)=c 0(x, y, z),
\end{array}\right.
$$

where $D_{x}, D_{y}, D_{z}$ are the constant heat diffusion coefficients, $\alpha_{x}, \alpha_{y}, \alpha_{z}$ are the constant mass transfer coefficients in the 3 kind boundary conditions, $c_{a z}, c_{a y}, c_{a x}$ are the given concentration on the boundaries, $t_{f}$ is the final time, $c 0(x, y, z)$ is the given initial concentration.

\section{THE CAM WITH THE HYPERBOLIC TYPE INTEGRAL SPLINE APPROXIMATION IN Z-DIRECTION FOR THE 3-D PROBLEM}

For solving IBVP (1) for every $t>0$ using CAM we consider the following hyperbolic type spline approximation with respect to $Z$-direction $c(x, y, z, t)=c_{z}(x, y, t)+m_{z}(x, y, t) f_{z 1}+e_{z}(x, y, t) f_{z 2}$ with the following two fixed hyperbolic functions $f_{z 1}, f_{z 2}$ and parameter $a_{z}$ :

$$
\begin{aligned}
& f_{z 1}=\frac{0.5 L_{z} \sinh \left(a_{z}\left(z-0.5 L_{z}\right)\right)}{\sinh \left(0.5 a_{z} L_{z}\right)}, \\
& f_{z 2}=\frac{\cosh \left(a_{z}\left(z-0.5 L_{z}\right)\right)-A_{0 z}}{8 \sinh ^{2}\left(0.25 a_{z} L_{z}\right)},
\end{aligned}
$$

where

$$
A_{0 z}=\frac{\sinh \left(0.5 a_{z} L_{z}\right)}{0.5 a_{z} L_{z}}
$$

$c_{z}(x, y, t)=\left(L_{z}\right)^{-1} \int_{0}^{L_{z}} c(x, y, z, t) d z \quad$ is the averaged value, $a_{z}>0$ is the initial parameter (unknown). It can be seen if parameter $a_{z}$ tends to zero then in the limit case we get the integral parabolic spline from A. Buikis [1]. The unknown functions $m_{z}, e_{z}$ are determined from boundary conditions of (1) by $z=0, z=L_{z}$ : $d_{z} m_{z}-k_{z} e_{z}=0, m_{z}=p_{z} e_{z}, p_{z}=k_{z} / d_{z}$, $d_{z}=0.5 a_{z} L_{z} \operatorname{coth}\left(0.5 a_{z} L_{z}\right), k_{z}=0.25 a_{z} \operatorname{coth}\left(0.25 a_{z} L_{z}\right)$ $D_{z}\left(d_{z} m_{z}+k_{z} e_{z}\right)+\alpha_{z}\left(c_{z}+0.5 m_{z} L_{z}+e_{z} b_{z}-c_{a z}\right)=0$ where $b_{z}=\frac{\cosh \left(0.5 a_{z} L_{z}\right)-A_{0 z}}{8 \sinh ^{2}\left(0.25 a_{z} L_{z}\right)}$.

Therefore $e_{z}=\left(c_{a z}-c_{z}\right) / g_{z}$, $g_{z}=b_{z}+0.5 p_{z} L_{z}+\left(2 k_{z} D_{z}\right) / \alpha_{z}$.

Now the initial-boundary value $2-\mathrm{D}$ problem is in following form (2):

$$
\left\{\begin{array}{l}
\frac{\partial}{\partial x}\left(D_{x} \frac{\partial c}{\partial x}\right)+\frac{\partial}{\partial y}\left(D_{y} \frac{\partial c}{\partial y}\right)+a_{0 z}^{2}\left(c_{a z}-c_{z}\right)=\frac{\partial c_{z}}{\partial t}, \\
x \in\left(0, L_{x}\right), y \in\left(0, L_{y}\right), t \in\left(0, t_{f}\right), \\
\frac{\partial c_{z}(0, y, t)}{\partial x}=\frac{\partial c_{z}(x, 0, t)}{\partial y}=0, \\
D_{x} \frac{\partial c_{z}\left(L_{x}, y, t\right)}{\partial x}+\alpha_{x}\left(c_{z}\left(L_{x}, y, t\right)-c_{a x}\right)=0, \\
D_{y} \frac{\partial c_{z}\left(x, L_{y}, t\right)}{\partial y}+\alpha_{y}\left(c_{z}\left(x, L_{y}, t\right)-c_{a y}\right)=0, \\
c(x, y, 0)=c 0_{z}(x, y),
\end{array}\right.
$$

where $a_{0 z}^{2}=\left(2 D_{z} k_{z}\right) / L_{z} g_{z}$,

$$
c 0_{z}(x, y)=\left(L_{z}\right)^{-1} \int_{0}^{L_{z}} c 0(x, y, z) d z \text {. }
$$

\section{THE CAM FOR CORRESPONDING SPECIAL 1-D INITIAL- BOUNDARY VALUE PROBLEM}

For comparison, we consider the corresponding 1-D problem with the following parameters

$$
\begin{aligned}
& a_{z}=1.79, D_{x}=D_{y}=0, c=c(z, t), L_{z}=1, \alpha_{z}=\propto \approx 10^{7}, c 0=0, \\
& D_{z}=0.01, c_{a z}=1, t_{f}=200 .
\end{aligned}
$$

Then the analytical solution we can obtaine from the following Fourier series [2]:

$$
\begin{aligned}
& U(z, t)=c_{a z}\left(1-\frac{4}{\pi} \sum_{i=0}^{\infty} \frac{(-1)^{i}}{2 i+1} \exp \left(-D_{z} \lambda_{i}^{2} t\right) \cos \left(\lambda_{i} z\right)\right), \\
& z \in\left(0, L_{z}\right), t \in\left(0, t_{f}\right), \text { where } \lambda_{i}=((2 i+1) \pi) /\left(2 L_{z}\right) .
\end{aligned}
$$

For the avareged value $U v(t)=\left(L_{z}\right)^{-1} \int_{0}^{L_{z}} U(z, t) d z$ we have following series:

$$
U v(t)=c_{a z}\left(1-\frac{8}{\pi^{2}} \sum_{i=0}^{\infty} \frac{1}{2 i+1} \exp \left(-D_{z} \lambda_{i}^{2} t\right)\right), t \in\left(0, t_{f}\right) .
$$


Environment. Technology. Resources. Rezekne, Latvia Proceedings of the $13^{\text {th }}$ International Scientific and Practical Conference. Volume 2, 69-73

From (2) we have following initial-value problem for ODE:

$\left\{\begin{array}{l}a_{0 z}^{2}\left(c_{a z}-u z(t)\right)=\frac{\partial u z(t)}{\partial t}, \\ u z(0)=0, t \in\left(0, t_{f}\right),\end{array}\right.$

where $u z(t)=c_{z}(t)$.

The averaged spline solution is in following form $u z(t)=c_{a z}\left(1-\exp \left(-a_{0 z}^{2} t\right)\right)$ and $U s(z, t)=c_{z}(t)+m_{z}(t) f_{z 1}+e_{Z}(t) f_{z 2}$

The numerical results with Matlab are obtained by $\propto \approx 20$ in the uniform grid

$z_{m}=m \cdot h_{z}, m=\overline{0, N_{z}}, h_{z}=L_{z} / N_{z}$,

$t_{k}=k \cdot h_{t}, k=\overline{0, N_{t}}, h_{t}=t_{f} / N_{t}, N_{z}=N_{t}=20$.

In the following figures (Fig.1.-Fig. 4.) there are represented the solutions $U(z, t), U s(z, t), U v(z, t)$, $u z(t)$.

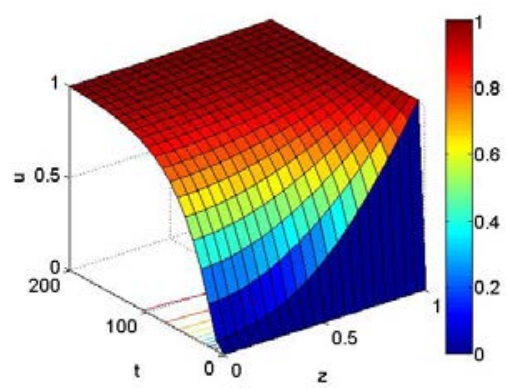

Fig. 1. Fourier series solution $U(z, t)$.

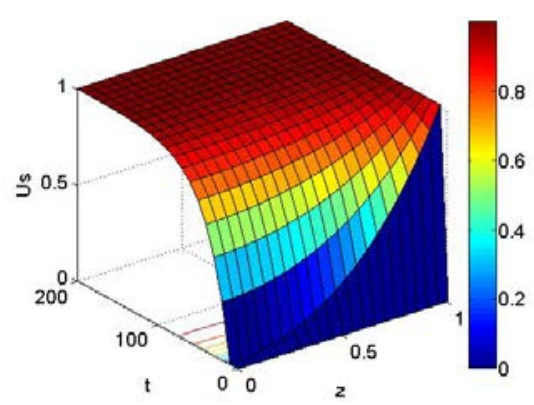

Fig. 2. Spline solution $U s(z, t)$.

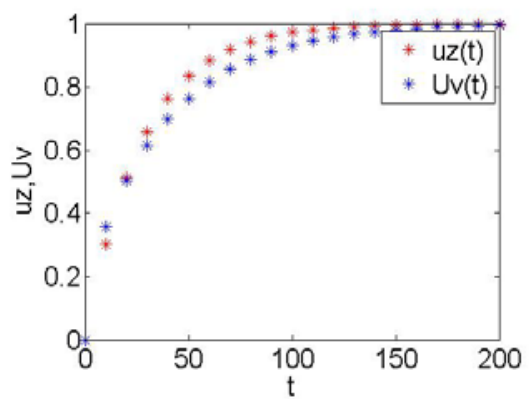

Fig. 3. Comparison the averaged solutions $u z(t)$ and $U v(t)$.

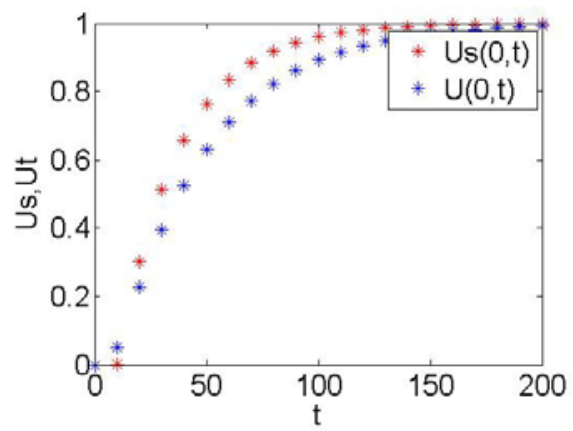

Fig. 4. Comparison the solutions $U(0, t)$ and $U s(0, t)$.

\section{THE CAM IN Y-DIRECTION FOR THE 2-D PROBLEM}

Using averaged method with respect to $y$ we apply $c_{y}(x, t)=\left(L_{y}\right)^{-1} \int_{0}^{L_{y}} c_{z}(x, y, t) d y$.

For the following hyperbolic type spline approximation $c_{z}(x, y, t)=c_{y}(x, t)+m_{y}(x, t) f_{y 1}+e_{y}(x, t) f_{y 2}$, we have

$$
\begin{aligned}
& f_{y 1}=\frac{0.5 L_{y} \sinh \left(a_{y}\left(y-0.5 L_{y}\right)\right)}{\sinh \left(0.5 a_{y} L_{y}\right)}, \\
& f_{y 2}=\frac{\cosh \left(a_{y}\left(y-0.5 L_{y}\right)\right)-A_{0 y}}{8 \sinh ^{2}\left(0.25 a_{y} L_{y}\right)},
\end{aligned}
$$

where $A_{0 y}=\frac{\sinh \left(0.5 a_{y} L_{y}\right)}{0.5 a_{y} L_{y}}$ and as the parameter we choose $a_{y}=a_{0 z} \sqrt{1 / D_{y}}$.

Similarly, we determine the unknown functions $m_{z}, e_{z}$ from boundary conditions by $z=0, z=L_{z}$ and $e_{y}=\left(c_{a y}-c_{y}\right) / g_{y}$, $g_{y}=b_{y}+0.5 p_{y} L_{y}+\left(2 k_{y} D_{y}\right) / \alpha_{y}$, $m_{y}=p_{y} e_{y}, p_{y}=k_{y} / d_{y}, d_{y}=0.5 a_{y} L_{y} \operatorname{coth}\left(0.5 a_{y} L_{y}\right)$, $k_{y}=0.25 a_{y} \operatorname{coth}\left(0.25 a_{y} L_{y}\right)$, $b_{y}=\frac{\cosh \left(0.5 a_{y} L_{y}\right)-A_{0 y}}{8 \sinh ^{2}\left(0.25 a_{y} L_{y}\right)}$.

The initial-boundary value $1-\mathrm{D}$ problem is in the following form (4): 


$$
\left\{\begin{array}{l}
\frac{\partial}{\partial x}\left(D_{x} \frac{\partial c_{y}}{\partial x}\right)+a_{0 y}^{2}\left(c_{a y}-c_{y}\right)+a_{0 z}^{2}\left(c_{a z}-c_{y}\right)=\frac{\partial c_{y}}{\partial t}, \\
\frac{\partial c_{y}(0, t)}{\partial x}=0, D_{x} \frac{\partial c_{y}\left(L_{x}, t\right)}{\partial x}+\alpha_{x}\left(c_{y}\left(L_{x}, t\right)-c_{a x}\right)=0, \\
c_{y}(x, 0)=c_{0} y(x), x \in\left(0, L_{x}\right), t \in\left(0, t_{f}\right),
\end{array}\right.
$$

where $a_{0 y}^{2}=\left(2 D_{y} k_{y}\right) / L_{y} g_{y}$,

$c_{o y}(x)=\left(L_{y}\right)^{-1} \int_{0}^{L_{y}} c_{o z}(x, y) d y$.

\section{THE CAM IN X-DIRECTION FOR THE 1-D PROBLEM}

It is possible proceed an averaging also in $X$ - direction $c_{x}(t)=\left(L_{x}\right)^{-1} \int_{0}^{L_{x}} c_{y}(x, t) d x$.

For the following hyperbolic type spline approximation $c_{y}(x, t)=c_{x}(t)+m_{x}(t) f_{x 1}+e_{x}(t) f_{x 2}$ we have

$$
\begin{aligned}
& f_{x 1}=\frac{0.5 L_{x} \sinh \left(a_{x}\left(x-0.5 L_{x}\right)\right)}{\sinh \left(0.5 a_{x} L_{x}\right)}, \\
& f_{x 2}=\frac{\cosh \left(a_{x}\left(x-0.5 L_{x}\right)\right)-A_{0 x}}{8 \sinh ^{2}\left(0.25 a_{x} L_{x}\right)},
\end{aligned}
$$

where $A_{0 x}=\frac{\sinh \left(0.5 a_{x} L_{x}\right)}{0.5 a_{x} L_{x}}$,

and as the parameter we choose $a_{x}=\sqrt{\left(a_{0 z}^{2}+a_{0 y}^{2}\right) / D_{x}}$. Similarly, we determine the unknown functions $m_{x}, e_{x}$ from boundary conditions by $x=0, x=L_{x}$ and $e_{X}=\left(c_{a x}-c_{X}\right) / g_{X}$, $g_{x}=b_{x}+0.5 p_{x} L_{x}+\left(2 k_{x} D_{x}\right) / \alpha_{x}, m_{x}=p_{x} e_{X}$, $p_{x}=k_{x} / d_{x}, d_{x}=0.5 a_{x} L_{x} \operatorname{coth}\left(0.5 a_{x} L_{x}\right)$, $k_{x}=0.25 a_{x} \operatorname{coth}\left(0.25 a_{x} L_{x}\right), b_{x}=\frac{\cosh \left(0.5 a_{x} L_{X}\right)-A_{0 x}}{8 \sinh ^{2}\left(0.25 a_{x} L_{X}\right)}$.

From the problem (4) follows the initial problem of linear ODEs

$$
\left\{\begin{array}{l}
\frac{\partial c_{X}(t)}{\partial t}=a_{0 y}^{2}\left(c_{a y}-c_{X}(t)\right)+a_{0 z}^{2}\left(c_{a z}-c_{X}(t)\right)+ \\
a_{0 x}^{2}\left(c_{a x}-c_{X}(t)\right)=0, c_{x}(0)=c_{0 x}, t \in\left(0, t_{f}\right),
\end{array}\right.
$$

where $c_{0 x}=\left(L_{x}\right)^{-1} \int_{0}^{L_{x}} c_{0 x}(x) d x$.

The solution of this problem can be obtained with the classical methods.

For $c_{0}=0$ we have, $c_{X}(t)=\left(A_{0} / B_{0}\right)\left(1-\exp \left(-B_{0} t\right)\right)$, where $A_{0}=a_{0 y}^{2} c_{a y}+a_{0 z}^{2} c_{a z}+a_{0 x}^{2} c_{a x}$,
$B_{0}=a_{0 y}^{2}+a_{0 z}^{2}+a_{0 x}^{2}$. In the stationary case we have $c_{X}=A_{0} / B_{0}$.

For fixed $t=t_{f}$ follows:

$$
\begin{aligned}
& c_{y}\left(x, t_{f}\right)=c_{x}\left(t_{f}\right)+m_{x}\left(t_{f}\right) f_{x 1}+e_{x}\left(t_{f}\right) f_{x 2}, \\
& e_{x}\left(t_{f}\right)=\left(c_{a x}-c_{x}\left(t_{f}\right)\right) / g_{x}, \\
& m_{x}\left(t_{f}\right)=p_{x} e_{x}\left(t_{f}\right), \\
& c_{z}\left(x, y, t_{f}\right)=c_{y}\left(x, t_{f}\right)+m_{y}\left(x, t_{f}\right) f_{y 1}+e_{y}\left(x, t_{f}\right) f_{y 2} \\
& e_{y}\left(x, t_{f}\right)=\left(c_{a y}-c_{y}\left(x, t_{f}\right)\right) / g_{y}, \\
& m_{y}\left(x, t_{f}\right)=p_{y} e_{y}\left(x, t_{f}\right), \\
& c\left(x, y, 0, t_{f}\right)=c_{z}\left(x, y, t_{f}\right)+m_{z}\left(x, y, t_{f}\right) f_{z 1}+e_{z}\left(x, y, t_{f}\right) f_{z 2} \\
& e_{z}\left(x, y, t_{f}\right)=\left(c_{a z}-c_{z}\left(x, y, t_{f}\right)\right) / g_{z}, \\
& m_{z}\left(x, y, t_{f}\right)=p_{z} e_{z}\left(x, y, t_{f}\right) .
\end{aligned}
$$

Taking into account, $x=0, y=0, z=0$, we get the following formulas:

$$
\begin{aligned}
& c_{y}(0, t)=c_{x}(t)+m_{x}(t) f_{x 1}+e_{x}(t) f_{x 2}, \\
& e_{x}(t)=\left(c_{a x}-c_{x}(t)\right) / g_{x}, m_{x}(t)=p_{x} e_{x}(t), \\
& c_{z}(0,0, t)=c_{y}(0, t)+m_{y}(0, t) f_{y 1}+e_{y}(0, t) f_{y 2}, \\
& e_{y}(0, t)=\left(c_{a y}-c_{y}(0, t)\right) / g_{y}, m_{y}(0, t)=p_{y} e_{y}(0, t), \\
& c(0,0,0, t)=c_{z}(0,0, t)+m_{z}(0,0, t) f_{z 1}+e_{z}(0,0, t) f_{z 2}, \\
& e_{z}(0,0, t)=\left(c_{a z}-c_{z}(0,0, t)\right) / g_{z}, m_{z}(0,0, t)=p_{z} e_{z}(0,0, t)
\end{aligned}
$$

\section{RESULTS AND DISCUSSION}

We use uniform grid in the space

$((N+1) \times(M+1) \times(K+1))$ :

$\left\{\left(x_{j}, y_{i}, z_{k}\right), y_{i}=(i-1) h_{y}, x_{j}=(j-1) h_{x}, z_{k}=(k-1) h_{z}\right\}$,

$i=\overline{1, M+1}, j=\overline{1, N+1}, k=\overline{1, K+1}$,

$M \cdot h_{y}=L_{y}, N \cdot h_{x}=L_{x}, K \cdot h_{z}=L_{z}$.

For the time $t \in\left[0, t_{f}\right]$ we use the moments

$t_{n}=n \tau, n=\overline{0, N_{t}}, \tau \cdot N_{t}=t_{f}$.

The numerical results are obtained for

$$
\begin{aligned}
& D_{x}=D_{y}=3 \cdot 10^{-4}, D_{z}=10^{-3}, L_{z}=3, L_{x}=L_{y}=1, \\
& \alpha_{z}=\alpha_{x}=\alpha_{y}=\propto \approx 10^{7}, M=N=K=N_{t}=20,
\end{aligned}
$$

For determining the parameter $a_{z}$ in the stationary case we do the iteration process with applying also the CAM first in $\mathrm{y}$-direction and then in $\mathrm{z}$-direction.

In $y$-direction we have $c(x, y, z)=c_{y}(x, z)+m_{y}(x, z) f_{y 1}+e_{y}(x, z) f_{y 2} \quad$, where $c_{y}(x, z)=\left(L_{y}\right)^{-1} \int_{0}^{L_{y}} c(x, y, z) d y$ is the averaged value and $a_{y}=a_{0 z} \sqrt{1 / D_{y}}$ is the previous value. In z-directon $c_{y}(x, z)=c_{z}(x)+m_{z}(x) f_{z 1}+e_{z}(x) f_{z 2} \quad$ where 
$c_{z}(x)=\left(L_{z}\right)^{-1} \int_{0}^{L_{z}} c_{y}(x, z) d z$ and $a_{z}=a_{0 y} \sqrt{1 / D_{z}}$ is the new value for parameter $a_{z}$. We can obtain quickly conversion iteration process (with 5 iteration) for obtaining the parameters $a_{z}, a_{y}, a_{x}$ with initial value $a_{z}=1$. We have the stationary solution with $\tau=1, t_{f}=200$ and with the maximal error $10^{-4}$. The maximal error between the 1-D exact problem and the spline solutions is 0.01334 . The results of averaged solutions for $t_{f}=200$ and depending on $x$ and $t$ we can see in (Fig. 5., Fig. 6.)

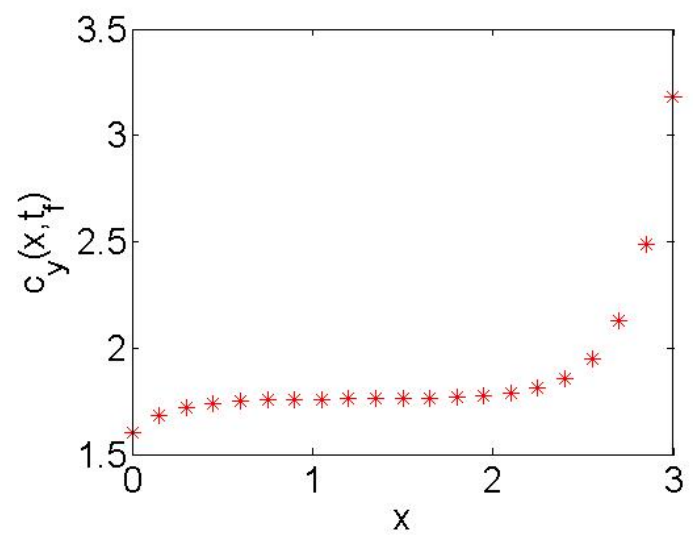

Fig. 5. The averaged solution $c_{y}\left(x, t_{f}\right)$.

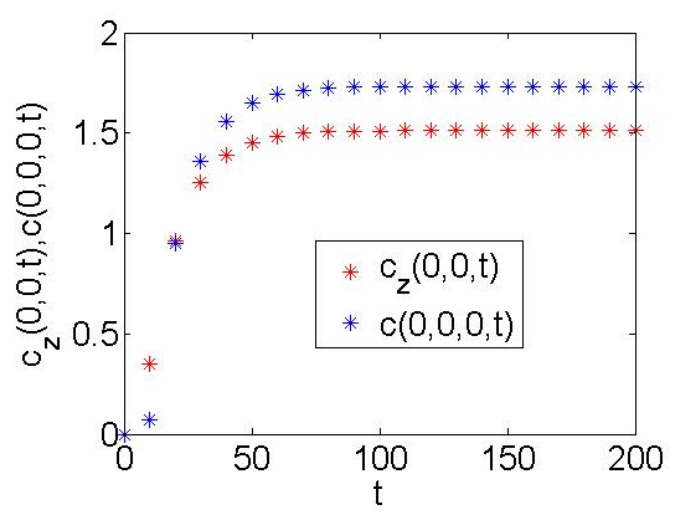

Fig. 6. The averaged solutions $C_{Z}(0,0, t)$ and $c(0,0,0, t)$.

\section{CONCLUSIONS}

1. In the present paper the conservative averaging method with special spline approximation is applied for solving the3-D non-stationary initial-boundary value (IBV) mass transfer problem.

2. This problem is reduced to 2-D and 1-D IBV problems using the integral hyperbolic type splines with fixed parameters.

3. Different orientation of the averaging allows you to determine the parameters of the spline function in such a way that the calculation error is minimal.

4. The solution of the special non-stationary 1-D IBV problem is obtained numerically using Fourier series method. This numerical solution is compared with the splaine function's solution and the maximal error is $10^{-4}$.

5. For testing the conservative averaging method also the exact solution of the 1-D IBV problem is found and the maximal error between the mentioned problem and the splaine function's solution, in this case, is 0.01334 .

\section{REFERENCES}

[1] A. Buikis, "The analysis of schemes for the modelling same processes of filtration in the underground, " in Acta Universitatis Latviensis, Vol. 592, Riga, 1994, pp. 25-32 (in Latvian).

[2] J. Crank, The matehematics of diffusion. Calewdon Press. Oxford, 1975.

[3] I. Kangro, H. Kalis, A. Gedroics, Ē. Teirumnieka, E. Teirumnieks, "On mathematical modelling of metals distribution in peat layers", "Mathematical Modelling and Analysis", vol. 19, issue 4, pp. 568588, 2014.

[4] E. Teirumnieka, I. Kangro E. Teirumnieks, H. Kalis, A. Gedroics, "The mathematical modeling of $\mathrm{Ca}$ and $\mathrm{Fe}$ distribution in peat layers", Proc. of the 8-th int. Scientific and Practical Conference "Environment. Technology. Resources", Rezekne Higher Education institution, June 20-22, volume 2, pp. 40-47, 2011.

[5] R. Vilums, A. Buikis, "Transient heat conduction in 3D fuse modelled by conservative averaging method", Topics in advanced theoretical and applied mechanics. Proceedings of International conference"3rd WSEAS International Conference on Applied and Theoretical Mechanics”, December 14-16, 2007, Puerto de la Cruz, Spain, pp. 54-63.

[6] R. Vilums, A. Rudevics "Cylindrical model of transient heat conduction in automotive fuse using conservative averaging method", Applied and computational mathematics, 2nd edition. Proceedings of International conference"13th WSEAS International Conference on Applied Mathematics”, December 1517, 2008, Puerto de la Cruz, pain, pp. 355-364. 